\title{
REMARKS ON THE CLIFFORD INDEX OF ALGEBRAIC CURVES
}

\author{
By
}

Katsumi AKAHORI

\begin{abstract}
Let $\operatorname{Cliff}(X)$ be the Clifford index of a curve $X$ and $L$ be a base-point-free line bundle on $X$ which satisfies $\operatorname{Cliff}(L)=$ $\operatorname{Cliff}(X)+k(k \geq 3)$. We determine sufficient conditions for the $|L|=g_{d}^{r}$ being simple (i.e. birationally very ample).
\end{abstract}

\section{Introduction}

Let $X$ be a smooth irreducible projective curve of genus $g \geq 4$ over an algebraically closed field of characteristic 0 and $L$ be a line bundle on $X$. A $g_{d}^{r}$ on $X$ be a linear series of degree $d$ and projective dimension $r$. If $|L|=g_{d}^{r}$, then the Clifford index of $L$ is defined by $\operatorname{Cliff}(L)=d-2 r$ and the Clifford index of $X$ is defined by $\operatorname{Cliff}(X)=\operatorname{Min}\{\operatorname{Cliff}(L) \mid r \geq 1, g-1-d+r \geq 1\}$. We say that a $|L|=g_{d}^{r}$ on $X$ computes $\operatorname{Cliff}(X)$ if $r \geq 1, g-1-d+r \geq 1$, and $\operatorname{Cliff}(X)=$ $d-2 r$. The following result about $\operatorname{Cliff}(X)$ is known:

Proposition 1.1 ([4]). Let a $|L|=g_{d}^{r}$ on $X$ compute $\operatorname{Cliff}(X), r \geq 3$. Then the $g_{d}^{r}$ is simple (i.e. birationally very ample) unless $X$ is hyperelliptic or bielliptic.

Furthermore, the same type results in the case of $\operatorname{Cliff}(L)=\operatorname{Cliff}(X)+k$ $(k=1,2)$ are known. Those results are as follows:

Proposition $1.2([2],(2.2))$. Let $a|L|=g_{d}^{r}$ be a base-point-free linear series on $X$. Assume that $\operatorname{Cliff}(L)=\operatorname{Cliff}(X)+1, \quad \operatorname{Cliff}(X) \geq 1, r \geq 3$, and $g-1-d+r \geq 1$. Then the $g_{d}^{r}$ is simple.

2000 Mathematics Subject Classification. 14H45, 14H51.

Key words and phrases. Clifford index, linear series.

Received May 16, 2006. 
Proposition 1.3 ([2], (2.3)). Let a $|L|=g_{d}^{r}$ be a base-point-free linear series on $X$. Assume that $\operatorname{Cliff}(L)=\operatorname{Cliff}(X)+2, \quad \operatorname{Cliff}(X) \geq 1, \quad r \geq 3$, and $g-1-d+r \geq 1$. Then the $g_{d}^{r}$ is simple unless $X$ and the $g_{d}^{r}$ are the following cases:

(1) $X$ is a trigonal curve, $\operatorname{Cliff}(X)=1$, and $|L|=g_{9}^{3}$;

(2) $X$ is a triple covering of an elliptic curve, $\operatorname{Cliff}(X)=4$, and $|L|=g_{12}^{3}$;

(3) $X$ is a double covering of a curve of genus $g(Y)=2,3,4,5,10$.

Our main purpose in this paper is to generalize the propositions stated above, that is to say, to describe $\operatorname{Cliff}(X)$ and $|L|=g_{d}^{r}$ which satisfy $\operatorname{Cliff}(L)=$ $\operatorname{Cliff}(X)+k(k \geq 3)$. We get the same type consequences for the case of $k=3,4$. Our results are the following:

THEOREM 1.4. Let a $|L|=g_{d}^{r}$ be a base-point-free linear series on $X$ of genus $g \geq 16$. Assume that $\operatorname{Cliff}(L)=\operatorname{Cliff}(X)+3, r \geq 3, g-1-d+r \geq 1$. Then the $g_{d}^{r}$ is simple unless $X$ and the $g_{d}^{r}$ are the following cases:

(1) $X$ is a trigonal curve, $\operatorname{Cliff}(X)=1$, and $|L|=g_{12}^{4}=4 g_{3}^{1}$;

(2) $X$ is a triple covering of an elliptic curve, $\operatorname{Cliff}(X)=4$, and $|L|=g_{15}^{4}$;

(3) $X$ is a double covering of a curve and $\operatorname{Cliff}(X)$ is odd.

THEOREM 1.5. Let a $|L|=g_{d}^{r}$ be a base-point-free linear series on $X$ of genus $g \geq 16$. Assume that $\operatorname{Cliff}(L)=\operatorname{Cliff}(X)+4, r \geq 3, g-1-d+r \geq 1$. Then the $g_{d}^{r}$ is simple unless $X$ and the $g_{d}^{r}$ are the following cases:

(1) $X$ is a 4-gonal curve, $\operatorname{Cliff}(X)=2$, and $|L|=g_{12}^{3}$;

(2) $X$ is a 4-sheeted covering of an elliptic curve, $\operatorname{Cliff}(X)=6$, and $|L|=g_{16}^{3}$;

(3) $X$ is a trigonal curve, $\operatorname{Cliff}(X)=1$, and $|L|=g_{15}^{5}$;

(4) $X$ is a triple covering of an elliptic curve, $\operatorname{Cliff}(X)=4$, and $|L|=g_{18}^{5}$;

(5) $X$ is a double covering of a curve and $\operatorname{Cliff}(X)$ is even.

In the case of $k \geq 5$, the result is similar to the preceding theorems.

THEOREM 1.6. Let a $|L|=g_{d}^{r}$ be a base-point-free linear series on $X$ of genus $g \geq 16$. Assume that $\operatorname{Cliff}(L)=\operatorname{Cliff}(X)+k(k \geq 5), r \geq k-1, g-1-d+r \geq$ 1. Then the $g_{d}^{r}$ is simple unless $X$ and the $g_{d}^{r}$ are the following cases:

(1) $X$ is a trigonal curve, $\operatorname{Cliff}(X)=1$, and $|L|=g_{3(k+1)}^{(k+1)}$;

(2) $X$ is a triple covering of an elliptic curve, $\operatorname{Cliff}(X)=4$, and $|L|=g_{3(k+2)}^{(k+1)}$;

(3) $X$ is a double covering of a curve and $\operatorname{Cliff}(X)+k$ is even. 
The organization of our paper is as follows. First we shall prove our theorems in $\S 2$. Next we shall derive $d \leq 3(\operatorname{Cliff}(X)+k)$ under some conditions from the main theorem in $\S 3$.

\section{On Linear Series $|L|$ with $\operatorname{Cliff}(L)=\operatorname{Cliff}(X)+k$}

We shall study about a linear series $g_{d}^{r}$ which satisfies $d-2 r=\operatorname{Cliff}(X)+k$ $(k \geq 0)$.

Proposition 2.1. Let a $|L|=g_{d}^{r} \quad$ on $\quad X \quad$ satisfy $\quad \operatorname{Cliff}(L)=\operatorname{Cliff}(X)+k$ $(k \geq 0), r \geq 3, g-1-d+r \geq 1$. Assume that the $g_{d}^{r}$ is base-point-free and defines a morphism $\pi: X \rightarrow \mathbf{P}^{\mathbf{r}}$, of degree $m \geq 2$ onto a curve $Y$ in $\mathbf{P}^{\mathbf{r}}$. If

$$
\operatorname{deg} \pi=m \geq \begin{cases}k / 2+2 & \text { for } k=2 l \\ (k-1) / 2+2 & \text { for } k=2 l+1,\end{cases}
$$

then $X$, Cliff $(X)$, and the $g_{d}^{r}$ are the following cases:

(a) if $k$ is even i.e. $k=2 l$, then

(i) $X$ is a $(l+2)$-gonal curve, $\operatorname{Cliff}(X)=l$, and

$$
|L|= \begin{cases}g_{2 r}^{r} & \text { for } l=0 \\ g_{3(l+2)}^{3} & \text { for } l>0\end{cases}
$$

(ii) $X$ is a $(l+2)$-sheeted covering of an elliptic curve, $\operatorname{Cliff}(X)=k+2$, and

$$
|L|= \begin{cases}g_{2(r+1)}^{r} & \text { for } l=0 \\ g_{4(l+2)}^{3} & \text { for } l>0\end{cases}
$$

(b) if $k$ is odd i.e. $k=2 l+1$, then

(i) $\pi: X \rightarrow Y \subset \mathbf{P}^{\mathbf{r}}$ is a $(l+2)$-sheeted covering of a rational curve $Y$, $\operatorname{Cliff}(X)=l-1$, and $|L|=g_{3(l+2)}^{3}=3 g_{(l+2)}^{1}$;

(ii) $k=3, X$ is a trigonal curve, $\operatorname{Cliff}(X)=1$, and $|L|=g_{12}^{4}=4 g_{3}^{1}$;

(iii) $k=3, X$ is a triple covering of an elliptic curve, $\operatorname{Cliff}(X)=4$, and $|L|=g_{15}^{4}$

(iv) $X$ is a $(l+2)$-sheeted covering of an elliptic curve, $\operatorname{Cliff}(X)=k$, and $|L|=g_{4(l+2)}^{3}$.

Proof. Let $c=\operatorname{Cliff}(X)$ and $d^{\prime}=\operatorname{deg} Y$. Then we have

$$
d^{\prime}=d / m=\{(c+k)+2 r\} / m
$$


and consider the induced complete linear series $g_{d^{\prime}}^{r}$ on the normalization of $Y$. If $g_{d^{\prime}}^{r}$ is not very ample, then there is a $g_{d^{\prime}-2}^{r-1}([3]$, IV. 3.1) on the normalization of $Y$. Hence $X$ admits a $g_{m\left(d^{\prime}-2\right)}^{r-1}$ and we get

$$
m\left(d^{\prime}-2\right)-2(r-1)=c+k-2(m-1) \leq \begin{cases}c-2 & \text { for } k=2 l \\ c-1 & \text { for } k=2 l+1 .\end{cases}
$$

This contradicts with the definition of $\operatorname{Cliff}(X)$. Therefore $g_{d^{\prime}}^{r}$ is very ample.

Thus $Y$ is a smooth non-degenerate and linearly normal curve of degree $d^{\prime}$ in $\mathbf{P}^{\mathbf{r}}$. We assume that $d^{\prime} \geq r+2$. Since it is well-known that any reduced irreducible and non-degenerate curve of degree $\geq r+2(r \geq 3)$ in $\mathbf{P}^{\mathbf{r}}$ has an $r$-secant- $(r-2)$-plane, we have a projection of $Y$ onto $\mathbf{P}^{\mathbf{1}}$ with center an $r$-secant$(r-2)$-plane, and we obtain a $g_{d^{\prime}-r}^{1}$ on $Y$. Hence there is a $g_{m\left(d^{\prime}-r\right)}^{1}$ on $X$ and we get

$$
m\left(d^{\prime}-r\right)-2=c+k-2-(m-2) r< \begin{cases}c-2 & \text { for } k=2 l \\ c-1 & \text { for } k=2 l+1 .\end{cases}
$$

This is a contradiction.

Therefore $Y$ in $\mathbf{P}^{\mathbf{r}}$ is the following 3 cases:

(1) $Y$ is a rational normal curve of degree $r$;

(2) $Y$ is a rational curve of degree $r+1$;

(3) $Y$ is an elliptic curve of degree $r+1$.

Case (1): In this case, $X$ has a $g_{m}^{1}$ and $d^{\prime}=r$. Hence we have

$$
m-2=\{(c+k)+2 r\} / r-2=(c+k) / r \geq c .
$$

By deriving from (I), we get $k \geq c(r-1)$. Since $r \geq 3$,

$$
c \leq \begin{cases}k / 2=l & \text { for } k=2 l \\ (k-1) / 2=l & \text { for } k=2 l+1 .\end{cases}
$$

First we shall consider the case of $k=2 l$. Let $c=k / 2=l$. We have $m-2=3 l / r \geq l$ by (I). If $l=0$, then $m=2$ for any $r$. If $l>0$, then $3 \geq r$. Since $r \geq 3$, we get $r=3$ and $m=l+2$. This case is (i) of (a). Let $c \leq l-1$. In this case, since $c+k \leq l-1+2 l=3 l-1<3 l$, we get $(c+k) / r<l$, whence $m<l+2$ by (I). This contradicts the assumption on $m$.

Next we shall consider the case of $k=2 l+1$. Let $c=(k-1) / 2=l$. We have $m-2=(3 l+1) / r \geq l$ by (I). Hence we get $l=1, r=4$, and $m=3$. This case is (ii) of (b). Let $c \leq l-1$, whence we obtain $(c+k) / r \leq 3 l / r \leq l$. On the other hand, we have $l \leq(c+k) / r$ by combining the assumption about $m$ with (I). Hence we get $(c+k) / r=l, m=l+2, r=3$, and $c=l-1$. This case is (i) of (b). 
Case (2): In this case, $X$ has a $g_{m}^{1}$ and $d^{\prime}=r+1$, whence we have

$$
m-2=\{(c+k)+2 r\} /(r+1)-2=(c+k-2) /(r+1) \geq c .
$$

By deriving from (II), we obtain $k-2 \geq c r \geq 0$. Since $r \geq 3$, we get $c \leq(k-2) / 3 \leq(k-2) / 2$. By using (II) again, we have $m-2 \leq$ $\{(k-2) / 2+k-2\} /(r+1) \leq k / 2-1$. This contradicts the assumption about $m$.

Case (3): In this case, $X$ has a $g_{2 m}^{1}$ and $d^{\prime}=r+1$. Therefore we have

$$
2 m-2=2(c+k+2 r) /(r+1)-2=2(c+k-1+r) /(r+1) \geq c .
$$

By deriving from (III), we get $(c-2)(r-1) \leq 2 k$. Since $r \geq 3$, we have $c \leq k+2$.

First we shall consider the case of $k=2 l$. Let $c=k+2$. We have $m=2 k /(r+1)+2$. If $l=0$, then $m=2$ for any $r$. If $l>0$, then $m \leq 2 k /$ $(3+1)+2=k / 2+2$. By virtue of the assumption, we get $m=k / 2+2$ and $r=3$. This case is (ii) of (a). Let $c \leq k+1$. We have $m<k / 2+2$. This contradicts the assumption.

Next we shall consider the case of $k=2 l+1$. By the same way stated above, we have $m \leq k / 2+2$. Combining this with the assumption, we get $m=(k-1) /$ $2+2$ and $c \leq k+1$. If $c=k+1$, then $m=(k-1) / 2+2=(2 k-1) /(r+1)+2$, whence we obtain $k=3, r=4$, and $m=3$. This case is (iii) of (b). Let $c=k$. We have $m \leq(k-1) / 2+2$. By virtue of the assumption, we get $m=(k-1) / 2+2$ and $r=3$. This case is (iv) of (b). Let $c \leq k-1$. We have $m<(k-1) / 2+2$. This contradicts the assumption.

Needless to say, the case of $k=0$ in (2.1) coincides with (1.1). Relating to this case, we shall prove the following lemma needed later.

Lemma 2.2. Let a $|L|=g_{d}^{r}$ on $X$ compute $\operatorname{Cliff}(X), r \geq 2$. Assume that $g_{d}^{r}$ is not simple and defines a morphism $\pi: X \rightarrow \mathbf{P}^{\mathbf{r}}$, of degree $m \geq 2$ onto a curve $Y$ in $\mathbf{P}^{\mathbf{r}}$. Then $\operatorname{deg} \pi=m=2$.

Proof. Let $\operatorname{deg} Y=d^{\prime}$ and $\left|L_{0}\right|=g_{d^{\prime}}^{r}$ be the induced complete linear series on the normalization of $Y$. If $m \geq 3$, then we have $h^{0}\left(L\left(-\pi^{*} y\right)\right)=$ $h^{0}\left(\pi^{*}\left(L_{0}(-y)\right)\right) \geq h^{0}\left(L_{0}(-y)\right)=r \geq 2, \quad h^{1}\left(L\left(-\pi^{*} y\right)\right) \geq 2, \quad$ and $\operatorname{Cliff}\left(L\left(-\pi^{*} y\right)\right)$ $<\operatorname{Cliff}(X)$ for any $y \in Y$. This is a contradiction. Therefore $m=2$.

Here we shall provide the proof for the case of $k \geq 3$ by using (2.1). 
Proof of TheOrem 1.4. Thanks to (2.1), we have only to consider the four cases. If $X$ is both a hyperelliptic curve and a trigonal curve, then we have $g \leq 2$ by Castelnuovo's lemma ([1], p. 366, C-1). It is a contradiction. If $X$ is a trigonal curve, then we are in case (1). If $X$ is a triple covering of an elliptic curve and $\operatorname{Cliff}(X)=4$, then we are in case (2). Let $X$ be a triple covering of an elliptic curve and $\operatorname{Cliff}(X)=3$. Since we assume that $g \geq 16, X$ carries $g_{5}^{1}$ by virtue of [5]. Applying Castelnuovo's lemma ([1], p. 366, C-1), we get $g \leq 11$. It is a contradiction.

Proof of Theorem 1.5. Assume that the $g_{d}^{r}$ is not simple and defines a morphism $\pi: X \rightarrow \mathbf{P}^{r}$, of degree $m \geq 2$ onto a curve $Y$ in $\mathbf{P}^{r}$. Let $c=\operatorname{Cliff}(X)$ and $d^{\prime}=\operatorname{deg} Y$. Then we have

$$
d^{\prime}=d / m=\{(c+4)+2 r\} / m
$$

and consider the induced complete linear series $g_{d^{\prime}}^{r}$ on the normalization of $Y$. If $m \geq 4$, we get the case (1) and (2) by means of (2.1). Let $m=3$. If $g_{d^{\prime}}^{r}$ is not very ample, $X$ has a $g_{3\left(d^{\prime}-2\right)}^{r-1}$ and we get $3\left(d^{\prime}-2\right)-2(r-1)=c$. By virtue of $(2.2), X$ must be a double covering of a curve. This contradicts $m=3$. Hence $g_{d^{\prime}}^{r}$ is very ample and we can get $d^{\prime} \leq r+1$ by repeating the way used in (2.1).

Here we may use the same method stated in (2.1). If $Y$ is a rational normal curve of degree $r$, then

$$
3-2=(c+4+2 r) / r-2=(c+4) / r \geq c .
$$

Since $4 \geq c(r-1)$ and $r \geq 3$, we get $c \leq 2$. Let $c=0$. Since $X$ is both a hyperelliptic and a trigonal curve, we have $g \leq 2$ by ([1], p. 366, C-1). It is a contradiction. Let $c=1$. We get $r=5$ and we are in case (3). Let $c=2$. We have $1=5 / r \geq 2$ and it is a contradiction. If $Y$ is a rational curve of degree $r+1$, then

$$
3-2=(c+4+2 r) /(r+1)-2=(c+2) /(r+1) \geq c .
$$

Since $2 \geq r c$ and $r \geq 3$, we obtain $c=0$. Let $c=0$. We have $1=2 /(r+1) \geq 0$, whence $r=1$. This contradicts the assumption. If $Y$ is an elliptic curve of degree $r+1$, then

$$
2 \cdot 3-2=2(c+4+2 r) /(r+1)-2=2(c+3+r) /(r+1) \geq c .
$$

Since $(c-2)(r-1) \leq 8$ and $r \geq 3$, we get $c \leq 6$. Let $c=0$ (resp. $c=1)$. We get $r=1$ (resp. $r=2$ ). It is a contradiction. Let $c=2$. Since we assume that $g \geq 16>10, X$ carries $g_{4}^{1}$ by virtue of [5]. Applying ([1], p. 366, C-1), we get $g \leq 9$. It is a contradiction. Let $c=3$. By the same way, we obtain $g \leq 11$ and 
this is a contradiction. Let $c=4$. We obtain $r=5$ and we are in case (4). Let $c=5$ (resp. $c=6$ ). We have $4 \geq c=5$ (resp. $4 \geq c=6$ ). It is a contradiction. If $m=2$, then we are in (5).

Proof of Theorem 1.6. Assume that the $g_{d}^{r}$ is not simple and defines a morphism $\pi: X \rightarrow \mathbf{P}^{r}$, of degree $m \geq 2$ onto a curve $Y$ in $\mathbf{P}^{r}$. Let $c=\operatorname{Cliff}(X)$ and $d^{\prime}=\operatorname{deg} Y$. Then

$$
d^{\prime}=d / m=\{(c+k)+2 r\} / m
$$

and consider the induced complete linear series $g_{d^{\prime}}^{r}$ on the normalization of $Y$. We shall prove this theorem by induction on $k(\geq 5)$.

First let $k=5$. If $m=2$, then we are in case (3). If $m \geq 4$, thanks to (2.1) there doesn't exist the $g_{d}^{r}$ which satisfies our condition. Let $m=3$. If $g_{d^{\prime}}^{r}$ is not very ample, $X$ has a $g_{3\left(d^{\prime}-2\right)}^{r-1}$ and $3\left(d^{\prime}-2\right)-2(r-1)=c+1$. By applying the results of the case of $k \leq 1, X$ has no complete linear series $|M|$ which satisfies $\operatorname{Cliff}(M) \leq c+1$. It is a contradiction. Therefore the $g_{d^{\prime}}^{r}$ is very ample and we may apply the same method used in (2.1). If $\operatorname{deg} Y \geq r+2$, then we have a projection of $Y$ onto $\mathbf{P}^{1}$ with center an $r$-secant- $(r-2)$-plane and obtain a $g_{d^{\prime}-r}^{1}$ on $Y$. Therefore there is a $g_{3\left(d^{\prime}-r\right)}^{1}$. Since $r \geq 4$, we get

$$
3\left(d^{\prime}-r\right)-2=c+3-r \leq c-1<c .
$$

This is a contradiction, whence $\operatorname{deg} Y \leq r+1$.

If $Y$ is a rational normal curve of degree $r$, then

$$
3-2=(c+5+2 r) / r-2=(c+5) / r \geq c .
$$

Since $5 \geq c(r-1)$ and $r \geq 4$, we get $c \leq 1$. Let $c=0$. By using ([1], p. 366, C-1), we get $g \leq 2$. It is a contradiction. Let $c=1$. We get $r=6$ and we are in case (1).

If $Y$ is a rational curve of degree $r+1$, then

$$
3-2=(c+5+2 r) /(r+1)-2=(c+3) /(r+1) \geq c .
$$

Since $3 \geq r c$ and $r \geq 4$, we obtain $c=0$. Let $c=0$. We have $1=3 /(r+1) \geq 0$, whence $r=2$. This contradicts the assumption.

If $Y$ is an elliptic curve of degree $r+1$, then

$$
2 \cdot 3-2=2(c+5+2 r) /(r+1)-2=2(c+4+r) /(r+1) \geq c .
$$

Since $(c-2)(r-1) \leq 10$ and $r \geq 4$, we get $c \leq 5$. Let $c=0$ (resp. $c=1)$. We get $r=2$ (resp. $r=3$ ). It is a contradiction. Let $c=2$ (resp. $c=3)$. By the same way we stated in the proof of $(1.5)$, we get $g \leq 9$ (resp. $g \leq 11$ ). It is a contradiction. 
Let $c=4$. We get $r=6$ and we are in case (2). Let $c=5$. We have $4 \geq c=5$. It is a contradiction.

Next let $k \geq 6$. If $m=2$, then we are in case (3). If

$$
m \geq \begin{cases}k / 2+2=l+2, & \text { for } k=2 l \\ (k-1) / 2+2=l+2, & \text { for } k=2 l+1,\end{cases}
$$

by virtue of (2.1) there doesn't exist the $g_{d}^{r}$ which satisfies our condition. Let $m=3$. If $g_{d^{\prime}}^{r}$ is not very ample, $X$ has $g_{3\left(d^{\prime}-2\right)}^{r-1}$ and $3\left(d^{\prime}-2\right)-2(r-1)=$ $c+k-4$. Here we may apply the results of the case of $k \leq 5$ and the induction hypothesis to the complete linear series $|N|$ which satisfies $\operatorname{Cliff}(N) \leq c+k-4$. Since we assumed the $X$ is a triple covering of a curve, we obtain $r-1 \leq$ $(k-4)+1=k-3$. This contradicts $r-1 \geq k-2$. Let $4 \leq m \leq l+1$. If $g_{d^{\prime}}^{r}$ is not very ample, we get a contradiction by the same way. Therefore the $g_{d^{\prime}}^{r}$ is very ample and we can repeat the way stated in the the case of $k=5$. If $\operatorname{deg} Y \geq r+2$, by virtue of a projection of $Y$ onto $\mathbf{P}^{\mathbf{1}}$ with center an $r$-secant$(r-2)$-plane we obtain a $g_{d^{\prime}-r}^{1}$ on $Y$ and a $g_{m\left(d^{\prime}-r\right)}^{1}$ on $X$. Since $m \geq 3$ and $r \geq k-1$, we get

$$
m\left(d^{\prime}-r\right)-2=c+k-2-(m-2) r \leq c+k-2-(3-2) \cdot(k-1)=c-1<c .
$$

Since this contradicts the definition of $\operatorname{Cliff}(X)$, we have deg $Y \leq r+1$.

If $Y$ is a rational normal curve of degree $r$, then

$$
m-2=(c+k+2 r) / r-2=(c+k) / r \geq c .
$$

Since $k \geq c(r-1)$ and $r \geq k-1$, we get $c \leq 1$. Let $c=0$. We get $r=k, m=3$. Applying ([1], p. 366, C-1), we get $g \leq 2$. It is a contradiction. Let $c=1$. We get $r=k+1, m=3$, and we are in case (1).

If $Y$ is a rational curve of degree $r+1$, then

$$
m-2=(c+k+2 r) /(r+1)-2=(c+k-2) /(r+1) \geq c .
$$

Since $k-2 \geq c r$ and $r \geq k-1$, we obtain $c=0$. Therefore we have $1 \leq m-2=$ $(k-2) /(r+1)$, whence $r \leq k-3$. This contradicts $r \geq k-1$.

If $Y$ is an elliptic curve of degree $r+1$, then

$$
2 m-2=2(c+k+2 r) /(r+1)-2=2(c+k-1+r) /(r+1) \geq c .
$$

Since $(c-2)(r-1) \leq 2 k$ and $r \geq k-1$, we get $c \leq 5$. Let $c=0$ (resp. $c=1)$. We have $2 \leq(k-1+r) /(r+1)$ (resp. $2 \leq(k+r) /(r+1)$ ), whence $r \leq k-3$ (resp. $r \leq k-2$ ). These contradicts the assumption. Let $c=2$ (resp. $c=3$ ). We get $(r, m)=(k-1,3)$ (resp. $(r, m)=(k, 3))$. But the same reason we stated above 
in the case of $k=5$, we can omit these cases. Let $c=4$. We obtain $2 \leq$ $(k+r+3) /(r+1)$, whence $r=k+1, m=3$, and we are in case (2). Let $c=5$. We get $2 \leq(k+r+4) /(r+1)$, whence $r=k+2$ and $m=3$. But this contradicts $2(m-1) \geq c$.

Furthermore, if $d$ is odd and $r \geq k+2$, then we can provide a sufficient condition for $g_{d}^{r}$ being simple by using (1.2), (1.3), (1.4), (1.5), and (1.6).

COROLlaRY 2.3. Let a $|L|=g_{d}^{r}$ be a base-point-free linear series on $X$ of genus $g \geq 16$. Assume that $\operatorname{Cliff}(L)=\operatorname{Cliff}(X)+k(k \geq 1), g-1-d+r \geq 1$. If $d$ is odd and $r \geq k+2$, then $g_{d}^{r}$ is simple.

\section{Some Corollaries}

In this section, we shall derive some corollaries from (2.3).

COROLlaRy 3.1. Let $a|L|=g_{d}^{r}$ be a base-point-free linear series on $X$ of genus $g \geq 16$. Assume that $\operatorname{Cliff}(L)=\operatorname{Cliff}(X)+k(k \geq 1)$ and $g-1-d+r \geq 1$. If $d$ is odd, $\operatorname{Cliff}(X) \geq 2 k+1$, and

$$
3(\operatorname{Cliff}(X)+2) / 2+2 k<d<2(\operatorname{Cliff}(X)+2),
$$

then we have $g \leq 2(\operatorname{Cliff}(X)+2+k)$.

Proof. Let $c=\operatorname{Cliff}(X)$. If $d \geq g$, then this is trivial. Therefore we may assume $d \leq g-1$. Since $d>3(c+2) / 2+2 k$ and $c \geq 2 k+1$, we get $2 r=d-c-$ $k>2(k+1)$, whence $r \geq k+2$. By using (2.3), we have $g_{d}^{r}$ is simple and $X$ may be regarded as a curve of degree $d$ in $\mathbf{P}^{\mathbf{r}}$. Assume that $X$ lies on a quadric $\mathbf{Q}$ in $\mathbf{P}^{\mathbf{r}}$ of rank $s \leq 4$. Then the two pencils of $(r-2)$-planes on $\mathbf{Q}$ sweep out pencil $g_{a}^{1}$ and $g_{b}^{1}$ on $X$ such that $a+b \leq d, a-2 \geq c$ and $b-2 \geq c$. This contradicts $d<2(c+2)$. Therefore the space of quadrics containing $X$ does not meet the closed subvariety of quadrics of rank $s \leq 4$ in $\mathbf{P}^{\mathbf{r}}$ which has codimension $(r-3)(r-2) / 2$ in the projective space of all quadrics in $\mathbf{P}^{\mathbf{r}}$. Hence we have $h^{0}\left(\mathbf{P}^{r}, I_{X}(2)\right) \leq(r-3)(r-2) / 2$ and

$$
\begin{aligned}
h^{0}\left(X, \mathcal{O}_{X}(2)\right) & \geq h^{0}\left(\mathbf{P}^{r}, \mathcal{O}(2)\right)-h^{0}\left(\mathbf{P}^{r}, I_{X}(2)\right) \\
& \geq(r+1)(r+2) / 2-(r-3)(r-2) / 2=4 r-2 .
\end{aligned}
$$

Since this means $h^{0}(2 L) \geq 4 r-2$, we get

$$
g=2 d+1-h^{0}(2 L)+h^{1}(2 L) \leq 2 d+1-(4 r-2)+h^{1}(2 L) .
$$


If $h^{1}(2 L) \leq 1$, then we have $g \leq 2(c+2+k)$. If $h^{1}(2 L) \geq 2$, then we have

$$
c \leq 2 d-2\left(h^{0}(2 L)-1\right) \leq 2 d-2(4 r-2)+2=4 c+4 k+6-2 d .
$$

This contradicts our hypothesis on $d$.

By applying the famous formula of Castelnuovo ([1], p. 116) to the result stated above, we get the following corollary.

COROLlaRy 3.2. Let a $|L|=g_{d}^{r}$ be a base-point-free linear series on $X$ of genus $g \geq 16$. Assume that $\operatorname{Cliff}(L)=\operatorname{Cliff}(X)+k(k \geq 1)$ and $g-1-d+r \geq 1$. If $d$ is odd, $d \leq g-1, \operatorname{Cliff}(X) \geq 2 k+1$, and $g \geq 3(\operatorname{Cliff}(X)+k+1)$, then

$$
d \leq 3(\operatorname{Cliff}(X)+2) / 2+2 k, \quad 2(\operatorname{Cliff}(X)+2) \leq d<2(\operatorname{Cliff}(X)+2+k) .
$$

Proof. Let $c=\operatorname{Cliff}(X)$. By virtue of (3.1), we may assume that $d \geq 2(c+2+k)$. Since $c \geq 2 k+1$, we have $2 r=d-c-k \geq c+k+4 \geq$ $2(k+1)+k+3>2(k+1)$ i.e. $r \geq k+2$, whence $g_{d}^{r}$ is simple by (2.3). We remark that $2(r-1)>c+k+1$. Hence we have $d-1=2(r-1)+c+k+1<$ $4(r-1)$. Therefore we see that $2<(d-1) /(r-1)<4$. Let $m=[(d-1) /(r-1)]$. In our case we have $m$ is 2 or 3 . Now we use Castelnuovo's bound ([1], p. 116).

If $m=2$, then we get $g \leq 2 d-3 r+1$. Since $[(d-1) /(r-1)]=$ $[\{c+k+1+2(r-1)\} /(r-1)]=2$, we have $c+k+1<r-1$, whence $d=$ $2 r+c+k<2 r+(r-2)=3 r-2<3 r-1 \leq 2 d-g$. Therefore $g<d$. It is a contradiction.

If $m=3$, then we get $g \leq 3(d-2 r+1)=3(c+k+1)$. Since $g \geq$ $3(c+k+1)$, we get $g=3(c+k+1)$, i.e. $X$ has the maximum possible genus. It is known that these curves of maximal genus have a $g_{4}^{1}([1]$, III, (2.6)). Therefore $\operatorname{Cliff}(X) \leq 2$. This contradicts $\operatorname{Cliff}(X) \geq 2 k+1$.

In [4] we find the result that $d \leq 3 \operatorname{Cliff}(X)$ for $\operatorname{Cliff}(X) \geq 3$. We shall present a similar type result in next corollary.

COROLlaRY 3.3. Let a $|L|=g_{d}^{r}$ be a base-point-free linear series on $X$ of genus $g \geq 16$. Assume that $\operatorname{Cliff}(L)=\operatorname{Cliff}(X)+k(k \geq 1)$ and $g-1-d+r \geq 1$. If $d$ is odd, $d \leq g-1$, and $\operatorname{Cliff}(X) \geq 2 k+1$, then we have $d \leq 3(\operatorname{Cliff}(X)+k)$.

Proof. Let $c=\operatorname{Cliff}(X)$. If $g \geq 3(c+k+1)$, then thanks to (3.2) we obtain $d \leq 2(c+k)+3 \leq 3(c+k)$. If $g \leq 3(c+k)+2$, then we have $d \leq g-1 \leq$ 
$3(c+k)+1$. But $d=3(c+k)+1$ means $d \not \equiv c+k(\bmod 2)$. Since $d-2 r=$ $c+k$, this is a contradiction.

\section{References}

[1] Arbarello, E., Cornalba, M., Griffiths, P. A., Harris, J., Geometry of algebraic curves, Vol. 1, Grundl. der math. Wiss. 267, Berlin-Heidelberg-New York 1985.

[2] Ballico, E., Keem, C., A remark on the Clifford index and higher order Clifford indices, J. Korean Math. Soc. 28 (1991), No. 1, 37-42.

[ 3 ] Hartshorne, R., Algebraic geometry, Grad. Texts in Math. 52, Berlin-Heidelberg-New York 1977.

[ 4 ] Keem, C., Kim, S., Martens, G., On a result of Farkas, J. reine angew. Math. 405 (1990), 112-116.

[5] Martens, G., Funktionen von vorgegebener Ordnung auf komplexen Kurven, J. reine angew. Math. 320 (1980), 68-85.

Department of Mathematics

Kyushu University

6-10-1 Hakozaki Higasi-ku

Fukuoka 812, Japan 